Article

\title{
Development of 30 Novel Polymorphic Expressed Sequence Tags (EST)-Derived Microsatellite Markers for the Miiuy Croaker, Miichthys miiuy
}

\section{Tianjun Xu, Dianqiao Sun, Yuena Sun and Rixin Wang *}

Key Laboratory for Marine Living Resources and Molecular Engineering, College of Marine Science, Zhejiang Ocean University, Zhoushan 316000, China; E-Mails: tianjunxu@163.com (T.X.); ssundianqiaod@163.com (D.S.); yuenasun@163.com (Y.S.)

* Author to whom correspondence should be addressed; E-Mail: wangrixin1123@126.com; Tel.: +86-580-2550826; Fax: +86-580-2550826.

Received: 17 February 2011; in revised form: 6 May 2011 / Accepted: 20 May 2011 / Published: 15 June 2011

\begin{abstract}
Expressed sequence tags (ESTs) can be used to identify microsatellite markers. We developed 30 polymorphic microsatellite markers from 5053 ESTs of the Miichthys miiuy. Out of 123 EST derived microsatellites for which PCR primers were designed, 30 loci were polymorphic in 30 individuals from a single natural population with 2-13 alleles per locus. The observed and expected heterozygosities were from 0.1024 to 0.7917 and from 0.2732 to 0.8845 , respectively. Nine loci deviated from the Hardy-Weinberg equilibrium, and linkage disequilibrium was significant between 22 pairs of loci. These polymorphic microsatellite loci will be useful for genetic diversity analysis and molecule-assisted breeding for M. miiuy.
\end{abstract}

Keywords: microsatellite; Expressed sequence tags (ESTs); Miichthys miiuy

\section{Introduction}

Miiuy croaker, Miichthys miiuy, is a promising marine fish species for culture in China and is distributed throughout eastern China ([1-3]. Although it is an important commercial fish species, little is known about the genetic information of miiuy croaker. There are no abundant molecular markers such as microsatellites isolated from this species. Lack of enough polymorphic molecular markers has 
limited development of molecular phylogeny, population structure, and conservation genetics and assisted selective breeding in this species. Thus, screening for polymorphic microsatellite or other molecular markers is necessary for analyzing genetic information in the miiuy croaker. Microsatellites are useful molecular markers to study population structure and genetic evolutionary information [4]. We have published 12 polymorphic microsatellite markers derived from two genomic libraries [5]. Up-to-date, only a few microsatellies markers are available for research in miiuy croaker.

There are many approaches for the development of microsatellite markers such as screening DNA or cDNA libraries for repeat motifs using hybridization and sequencing candidate clones [6], isolation from randomly amplified polymorphic DNA products [7], bioinformatic mining from database [8], etc. In general, the development of microsatellite markers has been limited by the labor and time required to construct, enrich, and sequence genomic libraries [9]. However, the development of microsatellite markers from expressed sequence tag (EST) database provides a rich source of valuable functional molecular markers. Herein, 30 polymorphic microsatellite markers were developed by bioinformatic mining EST sequences from M. miiuy.

\section{Materials and Methods}

We have constructed a normalized cDNA library from the spleen of the miiuy croaker. A total of 5053 ESTs from the library were sequenced [10]. The EST sequences were screened for mono-, di-, tri-, tetra-, penta-, and hexanucleotide repeats, 491 sequences contained repeat motifs. Primers for these partial loci were designed using PRIMER PREMIER 5.0 software (PREMIER Biosoft International, CA, USA). One hundred and twenty-three primer pairs were designed successfully. Some possessed only few repeats, which held less potential for useful polymorphism.

Genomic DNA was prepared from 30 individuals of miiuy croaker were captured from the Zhoushan fishing ground of the East China Sea. Total genomic DNA was extracted from gills using the TIANamp Genomic DNA Kit (Tiangen) following the manufacturer's instructions. PCR amplifications were carried out in $25-\mu \mathrm{L}$ volumes containing $2.5 \mu \mathrm{L}$ of $10 \times$ PCR buffer, $1.5 \mathrm{mM}$ $\mathrm{MgCl}_{2}, 0.2 \mathrm{mM} \mathrm{dNTP}, 0.2 \mu \mathrm{M}$ of the forward and reverse primers, and 1.5 units of Taq polymerase (Takara). Cycling conditions were $94{ }^{\circ} \mathrm{C}$ for 4 min followed by 30 cycles of $94{ }^{\circ} \mathrm{C}$ for $40 \mathrm{~s}$, annealing temperature for $45 \mathrm{~s}$ (see Table 1), and $72{ }^{\circ} \mathrm{C}$ for $40 \mathrm{~s}$, followed by 1 cycle of $72{ }^{\circ} \mathrm{C}$ for 5 min and then holding at $4{ }^{\circ} \mathrm{C}$. PCR amplification was performed on an ABI 9700 thermal cycler. Denatured amplified products were separated on $6 \%$ denaturing polyacrylamide (19:1 acrylamide:bis-acrylamide) gels using silver staining [6]. A denatured pBR322 DNA/MspI molecular weight marker (Tiangen) was used as a size standard to identify alleles. POPGENE32 [11] and ARLEQUIN 3.11 software [12] were used to calculate the number of alleles, observed $\left(H_{\mathrm{O}}\right)$ and expected $\left(H_{\mathrm{E}}\right)$ heterozygosity, violation of Hardy-Weinberg equilibrium (HWE) expectations and genotypic linkage disequilibrium. All results for multiple tests were corrected using sequential Bonferroni correction [13]. 
Table 1. Characterization of 30 polymorphic expressed sequence tags (EST)-derived microsatellite markers in M. miiuy.

\begin{tabular}{|c|c|c|c|c|c|c|c|c|c|c|}
\hline Locus & $\begin{array}{l}\text { GenBank } \\
\text { Accession No. }\end{array}$ & Repeat Motif & Gene & $\begin{array}{l}\text { Primer (5'-3') } \\
\text { [Forward (above) and Reverse (below)] }\end{array}$ & $\begin{array}{l}\text { Tm } \\
\left({ }^{\circ} \mathbf{C}\right)\end{array}$ & $\begin{array}{l}\text { No. of } \\
\text { Alleles }\end{array}$ & $\begin{array}{l}\text { Size range } \\
\text { (bp) }\end{array}$ & $\begin{array}{l}\text { No. of Null } \\
\text { Alleles }\end{array}$ & $\begin{array}{c}H_{\mathrm{O}} \\
\boldsymbol{H}_{\mathrm{E}}\end{array}$ & $P$-Value \\
\hline Mimi-4-C07 & GW668081 & $(\mathrm{GAA})_{5}$ & Ras-related protein Rab-35 & $\begin{array}{l}\text { TGAGGCACAATATGATGG } \\
\text { ACCGAGGACTTGGCTACT }\end{array}$ & 52 & 5 & $249-288$ & 1 & $\begin{array}{l}0.1481 \\
0.2732\end{array}$ & 0.0286 \\
\hline Mimi-5-B04 & GW668148 & $(\mathrm{AGTCAG})_{3}$ & unknown & $\begin{array}{l}\text { CTACCGCTGCTCTTCTGG } \\
\text { GATGGCTGGTCTACTTCG }\end{array}$ & 49 & 4 & $144-162$ & 0 & $\begin{array}{l}0.4286 \\
0.4662\end{array}$ & 0.0143 \\
\hline Mimi-5-G02 & GW668197 & $(\mathrm{AGA})_{5}$ & $\begin{array}{l}\text { NADH-cytochrome b5 } \\
\text { reductase } 2\end{array}$ & $\begin{array}{l}\text { TGTCCGTGCTGTTCTTCC } \\
\text { ATGGCTTATGTCCTGTTTCT }\end{array}$ & 49 & 5 & $157-169$ & 0 & $\begin{array}{l}0.2800 \\
0.3502\end{array}$ & 0.5507 \\
\hline Mimi-8-D03 & GW668391 & $(\mathrm{T})_{14}$ & unknown & $\begin{array}{l}\text { TTCAGTCAGGAGATTCAGGGTG } \\
\text { CAGCGGTTCAAACGGTCA }\end{array}$ & 48 & 6 & $119-128$ & 1 & $\begin{array}{l}0.4231 \\
0.7360\end{array}$ & 0.0020 \\
\hline Mimi-13-G10 & GW668718 & $(\mathrm{TTTG})_{5}$ & unknown & $\begin{array}{l}\text { GCGACAACGCAGACAGGA } \\
\text { CTTGGGCGGATGGTAGGA }\end{array}$ & 52 & 3 & $108-116$ & 0 & $\begin{array}{l}0.5217 \\
0.6309\end{array}$ & 0.1552 \\
\hline Mimi-16-A03 & GW668869 & $(\mathrm{T})_{15}$ & Cytochrome c & $\begin{array}{l}\text { TGGAGAACCCAAAGAAAT } \\
\text { CCACAAAGGAGCGTCATA }\end{array}$ & 52 & 7 & $282-297$ & 1 & $\begin{array}{l}0.3793 \\
0.8119\end{array}$ & $0.0000 *$ \\
\hline Mimi-16-E10 & GW668916 & $(\mathrm{TAGCT})_{5}$ & unknown & $\begin{array}{l}\text { GTTCTTTCACTGGCATCT } \\
\text { GCTGTTTCCACCTGTTTT }\end{array}$ & 50 & 6 & $189-224$ & 1 & $\begin{array}{l}0.4483 \\
0.6062\end{array}$ & 0.0262 \\
\hline Mimi-16-H01 & GW668939 & $(\mathrm{T})_{12}$ & unknown & $\begin{array}{l}\text { CAGTTGTGGGTTTGTTTG } \\
\text { TGTGGCGATGTTTCTTGT }\end{array}$ & 52 & 7 & $137-150$ & 1 & $\begin{array}{l}0.5909 \\
0.8478\end{array}$ & 0.0117 \\
\hline Mimi-21-G10 & GW669314 & $(\text { TTTAT })_{3}$ & $\begin{array}{l}\text { phosphatidic acid } \\
\text { phosphatase type } 2 \mathrm{~B}\end{array}$ & $\begin{array}{l}\text { GAGCGGGCTTTCCATTCA } \\
\text { TTCCCAAATCTGGTGTCTCG }\end{array}$ & 52 & 2 & $177-182$ & 1 & $\begin{array}{l}0.2222 \\
0.3522\end{array}$ & 0.0636 \\
\hline Mimi-28-G08 & GW669768 & $(\mathrm{A})_{14}$ & unknown & $\begin{array}{l}\text { GGGGAAGCACTTTATG } \\
\text { TCTTAGCGTGTTCTCGT }\end{array}$ & 52 & 5 & 199-203 & 1 & $\begin{array}{l}0.1538 \\
0.6380\end{array}$ & $0.0000 *$ \\
\hline Mimi-29-C05 & GW669810 & $(\mathrm{AGG})_{5} \ldots(\mathrm{T})_{16}$ & $\begin{array}{l}\text { similar to transmembrane } \\
\text { protein }\end{array}$ & $\begin{array}{l}\text { AGCCCTCСТCTGCTGTGA } \\
\text { CTGTTGCCTCCTGCСTGT }\end{array}$ & 52 & 5 & $119-126$ & 1 & $\begin{array}{l}0.2759 \\
0.5590\end{array}$ & 0.0311 \\
\hline Mimi-32-A10 & GW669955 & $(\mathrm{A})_{14} \mathrm{~N}_{12}(\mathrm{~T})_{17}$ & $\begin{array}{l}\text { Transmembrane protein } 32 \\
\text { precursor }\end{array}$ & $\begin{array}{l}\text { GAACCACCCATCCTTTTA } \\
\text { CTTTGCСCСТTCTGTCTA }\end{array}$ & 52 & 6 & $226-246$ & 1 & $\begin{array}{l}0.4348 \\
0.7739\end{array}$ & 0.0008 \\
\hline Mimi-32-B08 & GW669962 & $(\mathrm{A})_{14 \ldots} \ldots(\mathrm{T})_{14}$ & unknown & $\begin{array}{l}\text { CGTCGCACCAAGAATGAG } \\
\text { TGAAACCTACCGTCTACAAAT }\end{array}$ & 50 & 5 & $236-245$ & 1 & $\begin{array}{l}0.3846 \\
0.7398\end{array}$ & $0.0006 *$ \\
\hline Mimi-33-G06 & GW670085 & $(\mathrm{CT})_{10} \mathrm{~N}_{20}(\mathrm{CA})_{9}$ & unknown & $\begin{array}{l}\text { GGTAGGAGACTGGGTGGT } \\
\text { CAATGTTTCAGGCAAATGTA }\end{array}$ & 50 & 5 & $259-279$ & 1 & $\begin{array}{l}0.4815 \\
0.6723\end{array}$ & 0.0581 \\
\hline Mimi-34-A09 & GW670103 & $(\mathrm{A})_{13}$ & unknown & $\begin{array}{l}\text { TTTGGGTCACTAAATGGT } \\
\text { CGTCTGTAAAGCAGGTAA }\end{array}$ & 50 & 6 & $221-242$ & 1 & $\begin{array}{l}0.5172 \\
0.7992\end{array}$ & 0.0244 \\
\hline
\end{tabular}


Table 1. Cont.

\begin{tabular}{|c|c|c|c|c|c|c|c|c|c|c|}
\hline Mimi-35-E08 & GW670215 & $(\mathrm{T})_{12}$ & unknown & $\begin{array}{l}\text { ACGCACCCAACAACTCAG } \\
\text { ATGCTCATCTCCGCCTTA }\end{array}$ & 50 & 3 & $175-182$ & 1 & $\begin{array}{l}0.1923 \\
0.3288\end{array}$ & 0.0995 \\
\hline Mimi-36-C02 & GW670261 & $(\mathrm{TTTTC})_{3}$ & $\begin{array}{l}\text { ATPase, } \mathrm{Ca}^{++} \text {transporting, } \\
\text { plasma membrane 1a }\end{array}$ & $\begin{array}{l}\text { AATATCCCTGCCCTGCTA } \\
\text { TGTTCGCCATTGTCTTGC }\end{array}$ & 50 & 4 & $207-227$ & 1 & $\begin{array}{l}0.1034 \\
0.3575\end{array}$ & $0.0001 *$ \\
\hline Mimi-40-C05 & GW670563 & $(\mathrm{A})_{13}$ & unknown & $\begin{array}{l}\text { GTGTAACAAATAACCCTCG } \\
\text { TGCTGCTCGTCACAATAA }\end{array}$ & 50 & 4 & $131-143$ & 1 & $\begin{array}{l}0.4800 \\
0.7224\end{array}$ & 0.0152 \\
\hline Mimi-40-E05 & GW670585 & $(\mathrm{AAT})_{5}$ & Krueppel-like factor 6 & $\begin{array}{l}\text { AGGGCTCTGATCCATACA } \\
\text { TTCCGAAGTGCTCTACAA }\end{array}$ & 50 & 6 & $219-243$ & 1 & $\begin{array}{l}0.1333 \\
0.4418\end{array}$ & 0.0037 \\
\hline Mimi-40-H12 & GW670618 & $(\mathrm{CCT}) 5$ & unknown & $\begin{array}{l}\text { TCATCAGCACCAGCCTCT } \\
\text { CACATCCTCTTACСТCСТАTCT }\end{array}$ & 55 & 3 & $233-239$ & 0 & $\begin{array}{l}0.3704 \\
0.3934\end{array}$ & 0.0136 \\
\hline Mimi-41-E11 & GW670665 & $(\mathrm{GAA})_{5}$ & unknown & $\begin{array}{l}\text { ССТССТTCACCTCACCTT } \\
\text { ACATCTGTCCAGCCGTTT }\end{array}$ & 52 & 3 & $238-244$ & 1 & $\begin{array}{l}0.1379 \\
0.4120\end{array}$ & $0.0002 *$ \\
\hline Mimi-42-E04 & GW670734 & $(\mathrm{ATA})_{7}$ & $\begin{array}{l}\text { interleukin-8 receptor } \\
\text { CXCR1 }\end{array}$ & $\begin{array}{l}\text { CATTCATCACGGCTCCTT } \\
\text { TTCCCACTCTTATCTATCCA }\end{array}$ & 48 & 6 & $163-181$ & 0 & $\begin{array}{l}0.7200 \\
0.8196\end{array}$ & 0.1213 \\
\hline Mimi-42-G06 & GW670752 & $(\mathrm{TCC})_{6}$ & unknown & $\begin{array}{l}\text { TTGTTGTCTCGGTGATGG } \\
\text { GACTCCTGCTGTTGCTCC }\end{array}$ & 52 & 6 & $139-181$ & 0 & $\begin{array}{l}0.3750 \\
0.4787\end{array}$ & 0.4739 \\
\hline Mimi-43-H04 & GW670839 & $(\mathrm{TTTC})_{6}$ & unknown & $\begin{array}{l}\text { GCTTCCTGTCCCGTTTAT } \\
\text { TTTGCTCCCGTGGGTTAT }\end{array}$ & 52 & 13 & $141-217$ & 1 & $\begin{array}{l}0.6552 \\
0.8845\end{array}$ & 0.6188 \\
\hline Mimi-49-C10 & GW671186 & $(\mathrm{A})_{26}$ & eIF5A & $\begin{array}{l}\text { CGGCTTTACTTCAGTGGTT } \\
\text { TCTCCTCCTCGGTTGTCG }\end{array}$ & 54 & 7 & $180-190$ & 1 & $\begin{array}{l}0.4583 \\
0.8032\end{array}$ & 0.0192 \\
\hline Mimi-52-H10 & GW671455 & $\begin{array}{l}(\mathrm{GA})_{9}(\mathrm{CTGT})_{4} \ldots \\
(\mathrm{T})_{14}\end{array}$ & unknown & $\begin{array}{l}\text { ACGCATTTGTTTACTTTCTC } \\
\text { CACCACCATTCAGTTTCT }\end{array}$ & 50 & 4 & $188-202$ & 1 & $\begin{array}{l}0.4074 \\
0.7939\end{array}$ & $0.0001 *$ \\
\hline Mimi-54-A11 & GW671541 & $(\text { CTGGTC })_{6}$ & unknown & $\begin{array}{l}\text { AACCAAAGGGACCAAACG } \\
\text { GGAGCAGGCAGGTAAACG }\end{array}$ & 52 & 5 & $128-152$ & 0 & $\begin{array}{l}0.6207 \\
0.7042\end{array}$ & $0.0000 *$ \\
\hline Mimi-54-D06 & GW671567 & $(\mathrm{T})_{13} \ldots(\mathrm{A})_{15}$ & unknown & $\begin{array}{l}\text { TCСТCССАTACAAACTAA } \\
\text { GGTGGAAGACCGAAAA }\end{array}$ & 50 & 3 & $159-163$ & 0 & $\begin{array}{l}0.5769 \\
0.6750\end{array}$ & $0.0000 *$ \\
\hline Mimi-56-G05 & GW671751 & $(\mathrm{AGC})_{5}$ & unknown & $\begin{array}{l}\text { AGACACCCGACCAGAACC } \\
\text { ACAGCCTCCATCCACAAA }\end{array}$ & 54 & 4 & $154-160$ & 0 & $\begin{array}{l}0.7917 \\
0.6764\end{array}$ & 0.5599 \\
\hline Mimi-57-A05 & GW671772 & $(\mathrm{T})_{14}$ & unknown & $\begin{array}{l}\text { CTCCTGCCCTTCGTGATT } \\
\text { TCTTTCCCTGCTTGTTGTA }\end{array}$ & 50 & 6 & $113-133$ & 1 & $\begin{array}{l}0.1429 \\
0.4292 \\
\end{array}$ & $0.0011 *$ \\
\hline
\end{tabular}

$H_{\mathrm{O}}$ : Observed heterozygosity; $H_{\mathrm{E}}$ : Expected heterozygosity; Tm: Annealing temperature; * indicates significant deviation from HWE after Bonferroni correction $(P<0.0017)$. 


\section{Results and Discussion}

Details of the newly developed micorastellite loci and variability measures are summarized in Table 1. In total, 30 of 123 loci were successfully amplified and shown to be polymorphic in miiuy croaker. The number of alleles per locus ranging from two to thirteen, and observed and expected heterozygosities ranged from 0.1024 to 0.7917 and from 0.2732 to 0.8845 , respectively. The remaining 93 loci were no products or monomorphic in miiuy croaker. Nine loci significantly deviated from Hardy-Weinberg equilibrium in the sampled population after sequential Bonferroni correction $(P<0.0017)$, possibly due to the presence of null alleles, it is thought that these null alleles were caused by genetic instability within this region, the remaining 21 loci conformed to HWE. Further, null alleles were found in twenty-two loci (Table 1) and stuttering were found in nine loci (Mimi-16-A03, Mimi-21-G10, Mimi-28-G08, Mimi-29-C05, Mimi-32-B08, Mimi-36-C02, Mimi-40-E05, Mimi-41-E11, and Mimi-52-H10) detected with MICRO-CHECKER utility after Bonferroni correction [14], but no evidence for allelic dropout were found in any of the loci. In total, 24 pairwises (Mimi-16-E10 and Mimi-5-B04, Mimi-16-E10 and Mimi-13-G10, Mimi-16-E10 and Mimi-21-G10, Mimi-49-C10 and Mimi-21-G10, Mimi-5-B04 and Mimi-21-G10, Mimi-16-A03 and Mimi-21-G10, Mimi-16-H01 and Mimi-21-G10, Mimi-49-C10 and Mimi-32-A10, Mimi-16-H01 and Mimi-32-A10, Mimi-21-G10 and Mimi-32-A10, Mimi-32-A10 and Mimi-34-A09, Mimi-34-A09 and Mimi-35-E08, Mimi-4-C07 and Mimi-36-C02, Mimi-35-E08 and Mimi-40-H12, Mimi-36-C02 and Mimi-40-H12, Mimi-35-E08 and Mimi-41-E11, Mimi-36-C02 and Mimi-41-E11, Mimi-49-C10 and Mimi-54-D06, Mimi-32-A10 and Mimi-54-D06, Mimi-32-B08 and Mimi-54-D06, Mimi-35-E08 and Mimi-57-A05, Mimi-36-C02 and Mimi-57-A05, Mimi-40-H12 and Mimi-57-A05, Mimi-41-E11 and Mimi-57-A05) significant genotypic linkage disequilibrium were found among 285 pairs of the 30 loci after Bonferroni correction $(P<0.0017)$.

\section{Conclusions}

In the present study, 30 polymorphic microsatellite DNA markers were developed by cDNA sequences. These polymorphic microsatellite loci in miiuy croaker will enable studies of the genetic variation, population structure, conservation genetics and molecular assisted selective breeding of the miiuy croaker in the future.

\section{Acknowledgements}

This study was supported by Nation Nature Science Foundation of China (31001120), Zhejiang Provincial Natural Science Foundation of China (Y3100013) and Foundation of Zhejiang Educational Committee (Y200908463).

\section{References}

1. Zhang, Q.Y.; Hong, W.S. Status and prospects of artificial propagation and breeding technique of marine fish in China in the 1990s. Mod. Fish. Inform. 2000, 15, 3-6 (in Chinese).

2. Shan, X.J.; Cao, L.; Huang, W.; Dou, S.Z. Feeding, morphological changes and allometric growth during starvation in miiuy croaker larvae. Environ. Biol. Fishes 2008, 86, 121-130. 
3. Shan, X.J.; Xiao, Z.Z.; Huang, W.; Dou, S.Z. Effects of photoperiod on growth, mortality and digestive enzymes in miiuy croaker larvae and juveniles. Aquaculture 2008, 281, 70-76.

4. Liu, Y.G.; Liu, C.Y.; Li, F.Z.; Li, Z.X.; Wang, L. Development of microsatellite markers in sea perch, Lateolabrax Japonicus, from codominant amplified fragment length polymorphism bands. J. World Aquacult. Soc. 2009, 40, 522-530.

5. Wang, R.X.; Xu, T.J.; Sun, Y.N.; He, G.Y. Polymorphic microsatellite loci from two enriched genomic libraries for the genetic analysis of the miiuy croaker, Miichthys miiuy. Genet. Mol. Res. 2010, 9, 931-934.

6. Xu, T.J.; Shao, C.W.; Liao, X.L.; Ji, X.S.; Chen, S.L. Isolation and characterization of polymorphic microsatellite DNA markers in the rock bream (Oplegnathus fasciatus). Conserv. Genet. 2009, 10, 527-529.

7. Liu, Y.G.; Bao, B.L.; Liu, L.X.; Wang, L.; Lin, H. Isolation and characterization of polymorphic microsatellite loci from RAPD product in half-smooth tongue sole (Cynoglossus semilaevis) and a test of cross-species amplification. Mol. Ecol. Res. 2007, 8, 202-204.

8. Ji, X.S.; Chen, S.L.; Ma, H.Y.; Xu, T.J.; Liao, X.L.; Jiang, Y.L. Isolation and characterization of 19 EST-linked polymorphic microsatellite loci for olive flounder (Paralichthys olivaceus). Aquacult. Res. 2009, 40, 980-983.

9. Edwards, K.J.; Barker, J.H.A.; Daly, A.; Jones, C.; Karp, A. Microsatellite libraries enriched for several microsatellite sequences in plants. Biotechniques 1996, 20, 758-760.

10. Xu, T.J.; Meng, F.X.; Sun, Y.N.; Shi, G.; Wang, R.X. Identification of immune genes of the miiuy croaker (Miichthys miiuy) by sequencing and bioinformatic analysis of ESTs. Fish Shellfish Immunol. 2010, 29, 1099-1105.

11. Yeh, F.C.; Boyle, T.J.B. Population genetic analysis of co-dominant and dominant markers and quantitative traits. Belg. J. Bot. 1997, 129, 157.

12. Schneider, S.; Roessli, D.; Excoffier, L. ARLEQUIN: A Software for Population Genetics Date Analysis, Version 2.000; Genetics and Biometry Laboratory, Department of Anthropology, University of Geneva: Geneva, Switzerland, 2000.

13. Rice, W.E. Analyzing tables of statistical tests. Evolution 1989, 43, 223-225.

14. van Oosterhout, C.; Hutchinson, W.F.; Wills, D.P.M.; Shipley, P. MICRO-CHECKER: software for identifying and correcting genotyping errors in mirosatellite data. Mol. Ecol. Notes 2004, 4, $535-538$.

(C) 2011 by the authors; licensee MDPI, Basel, Switzerland. This article is an open access article distributed under the terms and conditions of the Creative Commons Attribution license (http://creativecommons.org/licenses/by/3.0/). 